\title{
Severe airflow obstruction and eosinophilic lung disease after Stevens-Johnson syndrome
}

\author{
A.P. Shah*, H. Xü ${ }^{\#}$ P.J. Sime* and D.R. Trawick*
}

ABSTRACT: Respiratory involvement is a frequent complication of Stevens-Johnson syndrome (SJS). However, there are very few convincing reports of persistent pulmonary sequelae, as demonstrated by spirometry, radiology and pathology.

The current study presents a case of a 13-yr-old female with T-cell acute lymphocytic leukaemia who developed persistent, severe, obstructive lung disease following an episode of SJS.

A lung biopsy demonstrated bronchiolar submucosal fibrosis consistent with constrictive bronchiolitis, as well as eosinophilic micro-abscesses, which, to the current authors' knowledge, has not been previously described.

The present study illustrates specific histopathological features that highlight a possible association between Stevens-Johnson syndrome, constrictive bronchiolitis and eosinophilic micro-abscesses. The eosinophils may be associated with permanent mucosal damage, as seen in the present case, by releasing mediators that have a pro-fibrogenetic role. However, further investigation is warranted.

KEYWORDS: Constrictive bronchiolitis, eosinophilic micro-abscesses, pulmonary disease, Stevens-Johnson syndrome

tevens-Johnson syndrome (SJS) is an acute self-limited eruption of the skin and mucus membranes, which represents a hypersensitivity reaction to various agents [1]. Although rare, pulmonary complications do occur with SJS [2]. The current study presents a patient who developed persistent and severe airflow obstruction with associated eosinophilic lung micro-abscesses following an episode of SJS.

\section{CASE REPORT}

A 13-yr-old Caucasian female with T-cell acute lymphocytic leukaemia underwent an allogeneic matched sibling bone-marrow transplant following a conditioning regimen of total body irradiation, cyclophosphamide and mesna without complications. On day 17, trimethoprim-sulphamethoxazole for Pneumocystis carinii (P. jiroveci) pneumonia (PCP) prophylaxis was initiated. On day 31, the patient developed a rash and severe mucositis. She was not hypoxic and lung examination demonstrated bilateral rhonchi without wheezing. She had scattered erythematous macules and papules with the greatest concentration on her superior chest, but also involving her oral mucosa, conjunctiva and urethra. Her palms and soles were unaffected. An ophthalmological consultation detailed ocular involvement with pseudomembranous formation. A skin biopsy of the patient's left shoulder 3 days after the onset of rash demonstrated individual and confluent epidermal necrosis consistent with erythema multiforme. The paucity of lymphocytes in the epidermis opposed a diagnosis of graft versus host disease (GVHD). The patient's presentation was consistent with SJS, probably due to trimethoprim-sulphamethoxazole.

Within the next 2 weeks, she experienced progressive dyspnoea on exertion, wheezing and a nonproductive cough with a nocturnal predilection, but without fever, chills or night sweats. Subsequent pulmonary function tests demonstrated the evolution of severe airflow obstruction relative to the pre-transplant tests (fig. 1), but a relatively preserved diffusion capacity for carbon monoxide. Chest radiography was unremarkable. The patient was discharged 52 days after the transplant.
AFFILIATIONS

Depts of *Medicine, Pulmonary and Critical Care Unit, and

\#Pathology and Laboratory Medicine, University of Rochester Medical Center, Rochester, NY, USA.

CORRESPONDENCE

A.P. Shah

601 Elmwood Avenue

Box 692

Rochester

NY 14586

USA

Fax: 15852731114

E-mail: alpashah@hotmail.com

Received:

March 132006

Accepted after revision:

July 102006

\section{SUPPORT STATEMENT}

There is no financial support from any organisation and there is no potential conflict of interest. 
Further work-up performed on an outpatient basis included a high-resolution computed tomography (HRCT) scan of the patient's chest, which demonstrated a mosaic pattern with ground-glass opacifications, mild central bronchiectasis (most prominent in the right-middle and right-lower lobes), and multiple nodular and linear branching densities (fig. 2). Bronchoscopy failed to demonstrate any anatomical abnormality, and the culture of bronchoalveolar lavage fluid only grew parainfluenzae. Video-assisted thoracic surgery (VATS), $\sim 3$ months after the onset of dyspnoea, demonstrated bronchiolar submucosal fibrosis consistent with constrictive bronchiolitis (CB; fig. 3). She also had type-2 pneumocyte hyperplasia and organising eosinophilic pneumonia-like areas. Of particular interest was the presence of eosinophils in the interstitium and in the alveolar spaces (fig. 4) forming focal eosinophilic microabscesses. The bronchiolocentric eosinophilic process is not a common pattern in eosinophilic pneumonia. Special stains and cultures for fungi, mycobacteria, pneumocystis, cytomegalovirus and adenovirus were all negative. CD1a and S-100 immunohistochemical stains for Langerhans' cells were also negative.

The patient was treated with high-dose glucocorticoids for $\mathrm{CB}$ for $\sim 1$ yr with some symptomatic relief, although her response to an albuterol metered-dose inhaler was variable. The
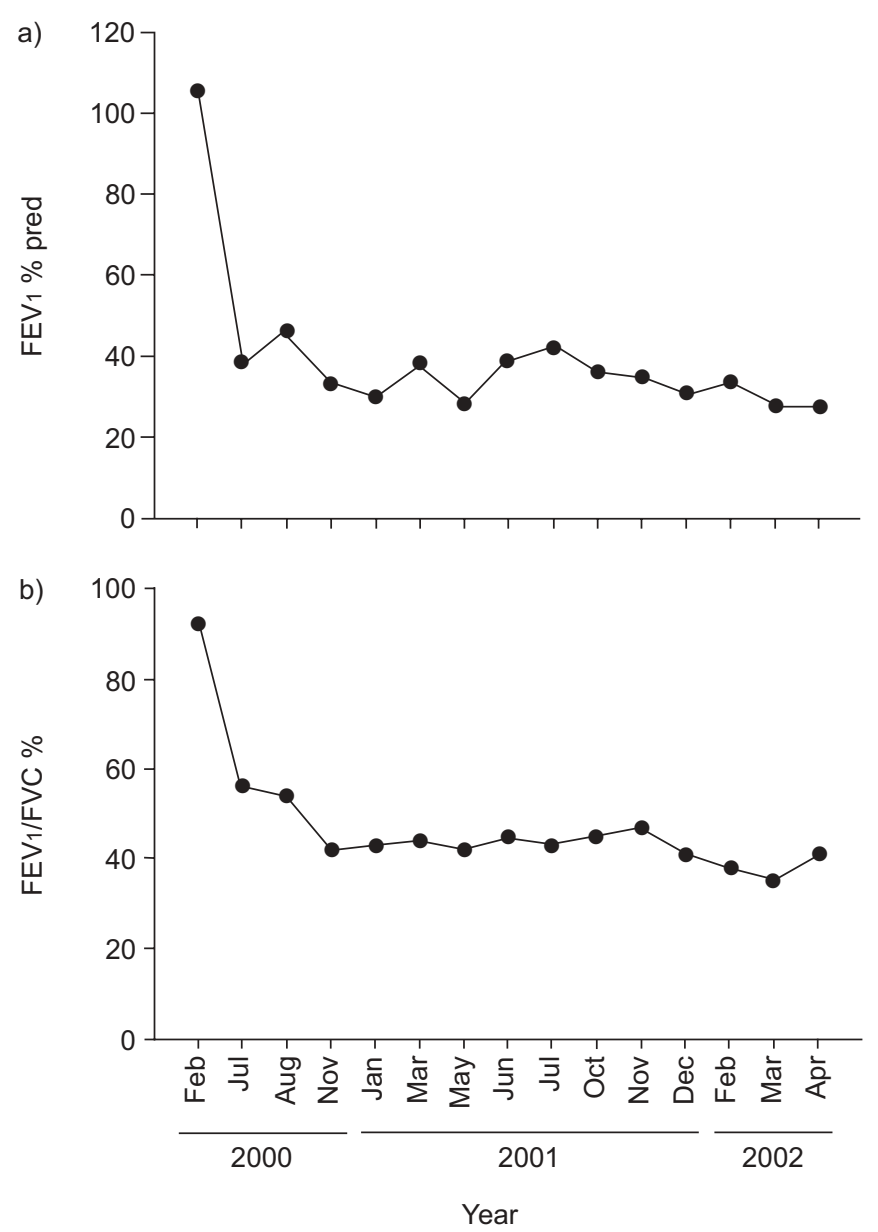

FIGURE 1. Cumulative spirometric values over a 26-month period, demonstrating the evolution and persistence of severe airflow obstruction. FEV 1 : forced expiratory volume in one second; FVC: forced vital capacity; \% pred: \% predicted.

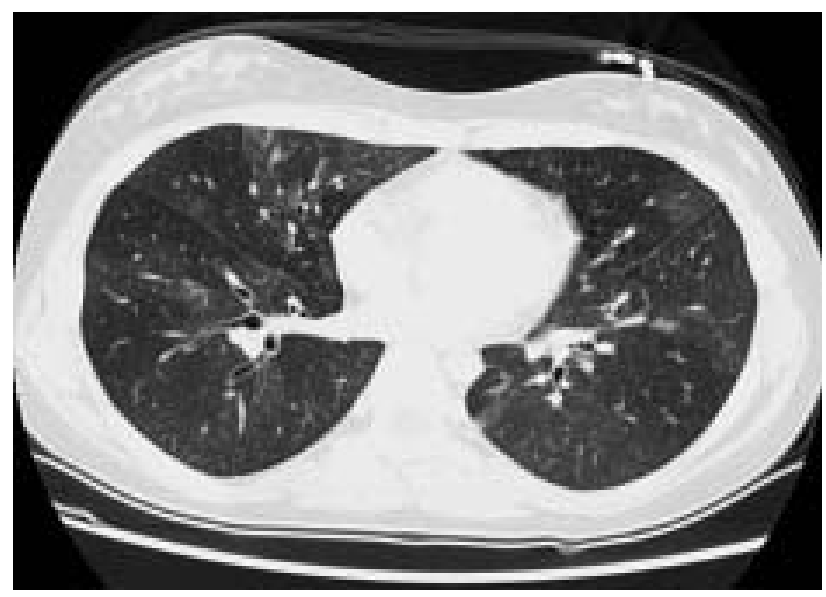

FIGURE 2. High-resolution computed tomography scan of the lower lobes showing a mosaic pattern with ground-glass opacifications, central bronchiectasis, multiple centrilobular nodules and linear branching densities, suggesting bronchiolar lesions.

glucocorticoids have since been slowly tapered and her airflow obstruction remained fixed for $\geqslant 2 \mathrm{yrs}$, as demonstrated by multiple pulmonary function tests.

\section{DISCUSSION}

The current study presents the case of a 13-yr-old female who developed SJS and, subsequently, experienced progressive airflow obstruction from CB. The most intriguing feature of this case, however, was the presence of eosinophilic microabscesses on lung pathology, which, to the current authors' knowledge, has not been described before in SJS.

SJS, first described in children in 1922, is associated with febrile erosive stomatitis, severe ocular involvement and a disseminated cutaneous eruption of discrete dark-red macules, sometimes with a necrotic centre [3]. Various underlying causes have been postulated [4]. The patient was diagnosed with SJS on the basis of her history, clinical appearance, ophthalmological examination and pathological findings. Her recent exposure to trimethoprim-sulfamethoxazole for PCP prophylaxis was the likely causative agent [3].

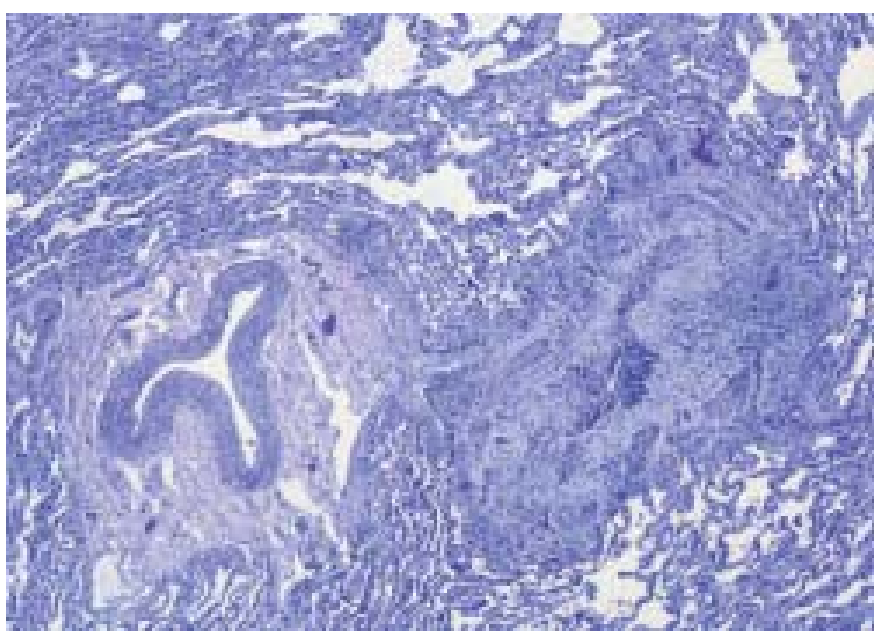

FIGURE 3. Video-assisted thoracoscopic lung biopsy of the left-upper lobe. The haematoxylin-eosin stain shows bronchiolar submucosal fibrosis consistent with constrictive bronchiolitis. 

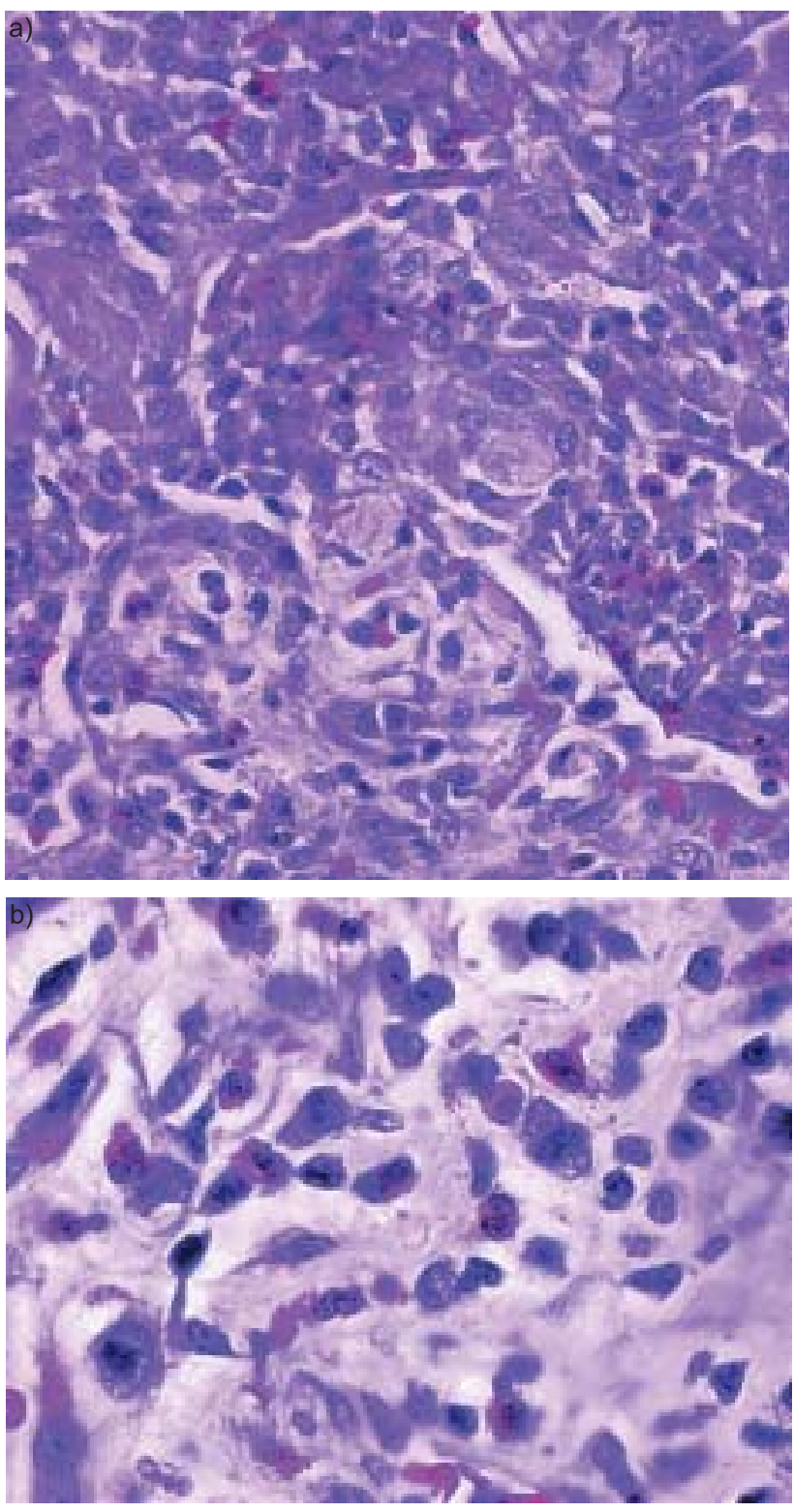

FIGURE 4. a) Video-assisted thoracoscopic lung biopsy of the left-upper lobe with haematoxylin-eosin staining. Eosinophils are present in the interstitium as well as the alveolar spaces, which are referred to as eosinophilic micro-abscesses. a) A high-power view demonstrating a closer look at the eosinophils.

Respiratory pathology in SJS occurs in up to $25-45 \%$ of cases, and may include mucosal involvement of the upper airway and respiratory tree, pneumonia, pneumothorax and mediastinal emphysema [5]. Up to $30 \%$ of cases are preceded by an atypical pneumonia and an association with Mycoplasma pneumoniae infection is well established [6]. Persistent pulmonary sequelae following recovery from SJS are rare. However, the incidence of complications may be higher, and the consequences more severe in toxic epidermal necrolysis, the more extensive form of SJS [1].

$\mathrm{CB}$, rarely reported in association with SJS, was evident on the patient's lung biopsy. Pathologically, CB is characterised by the distinctive pattern of a peribronchiolar fibrosing inflammatory process, resulting in complete obstructive scarring of the bronchiolar lumen leading to obliteration of the airway [7]. Physiologically, CB is associated with progressive airflow obstruction with severe air trapping and a variable response to bronchodilators, as was seen on the patient's pulmonary function tests. This pattern is consistent with significant involvement of the small intrapulmonary airways, due to the organisation of an inflammatory exudate resulting in fibrous narrowing or obliteration of these airways [6]. The patient's HRCT scan demonstrated a mosaic pattern with ground-glass opacifications, mild central bronchiectasis and multiple nodular (probably reflecting centrilobular prominence) and linear branching densities, which are consistent with features of $\mathrm{CB}$ [7] and were further evidenced in subsequent scans.

$\mathrm{CB}$ is associated with numerous conditions [7], including GVHD after lung and bone-marrow transplant [8]. This raises the possibility that this was the aetiology of the patient's CB. However, her clinical presentation and skin pathology do not support this hypothesis [9]. The patient received a six out of six antigen-match allogeneic bone-marrow transplant from her sister 1 month prior to development of SJS. She did not have any other peripheral signs or symptoms of GVHD. She presented with an erythematous maculo-papular rash that spared her palms and soles; GVHD usually affects both [9]. The involvement of at least two mucous membranes is one of the clinical features of SJS [3]. However, the patient had involvement of her oral cavity, conjunctiva and urethra. Conjunctival lesions occur in $\sim 85 \%$ of patients with SJS [10], and seromembrane formation on the conjunctiva (which was seen in the current patient) is a classic feature of SJS. In addition, on skin biopsy, the absence of significant numbers of lymphocytes in the epidermis did not support a diagnosis of GVHD. Laboratory tests performed at that time, including alkaline phosphatase and total bilirubin, were within normal limits. Also, a strong temporal association existed between SJS and the development of obstructive lung disease in the patient. In most patients with SJS who develop pulmonary pathology, the appearance of signs and symptoms occurs within 2-4 weeks after the onset of SJS [1, 11, 12]. Conversely, СВ associated with GVHD usually appears much later $[13,14]$, and is usually a manifestation of late disease in GVHD patients.

The histology result, obtained by a VATS lung biopsy, is the most intriguing and convincing piece of data that helps to categorise the aetiology of chronic lung disease in the patient. Eosinophils were seen both in the interstitium and in the alveolar spaces where they focally formed eosinophilic microabscesses, which are more consistent with the immunology of SJS, as it is an allergic-mediated lung disease. This may reflect, to some extent, its pathophysiological mechanisms.

The sequence of changes previously described in the upper airways of patients with SJS, such as mucosal ulceration and loss of airway epithelium and proliferation of granulation tissue in bronchi [15], may be reflected in the lower respiratory tract in the patient. The striking proliferation of type-2 pneumocytes with significant cytological atypia seen in the patient suggests that there was severe injury to both bronchiolar and alveolar epithelium and there were now prominent regenerative changes. 
The pathogenesis of chronic pulmonary sequelae in SJS is not well understood. One theory is that $\mathrm{CB}$ appears as a consequence of mucosal damage due to immune complex deposition in SJS. Another theory is that there may be a component of allergic inflammation, which is demonstrated by the presence of eosinophils. It is interesting to speculate on the pathological role of eosinophils in regulating the submucosal fibrosis and epithelial damage observed in the present case report. Eosinophils are certainly prominent in diseases where remodelling and fibrosis occur. These diseases include asthma and the associated bronchial wall remodelling, and patients with a variety of interstitial pulmonary fibroses [16]. Indeed, there are now significant data demonstrating that eosinophils are important sources of a variety of pro-fibrogenic mediators, including: transforming growth factor (TGF)- $\alpha$ [17]; TGF- $\beta$ [18]; vascular endothelial growth factor [19]; and type 2 cytokines, such as interleukin-13 [20]. When activated, eosinophils also release a variety of molecules that are toxic to the epithelium, including eosinophil cationic protein [21]. These mediators may be causally implicated in the mucosal damage observed in the current patient. It is well known that activated eosinophils can cause injury to major organ systems and damage epithelium due to infiltration, as well as mediator release, such as in the hypereosinophilic syndromes [22]. Thus, there are several mechanisms by which eosinophils may be implicated in the pulmonary manifestations of SJS. In the patient with SJS, the role of eosinophils may be important, but it is yet to be determined.

\section{Conclusion}

In conclusion, the current authors presented the case of a 13-yrold female with biopsy-proven SJS, who developed progressive and persistent airflow obstruction with classic features of CB obliterans as evidenced physiologically, radiologically and pathologically. The lung histopathology was the most intriguing feature of the case. There was the presence of eosinophilic micro-abscesses, which has not been previously described. The present authors believe that these microabscesses may provide greater insight into the pathophysiology of pulmonary disease associated with SJS.

Although the patient was discharged from hospital, her pulmonary disease has not responded to steroids during a 4yr follow-up. This represents permanent pulmonary sequelae of Stevens-Johnson syndrome in the absence of toxic epidermal necrolysis. The current authors speculate that eosinophilic micro-abscesses and constrictive bronchiolitis may be specific pathological findings associated with Stevens-Johnson syndrome, which warrant further investigation.

\section{REFERENCES}

1 Basker M, Cherian T, Raghupathy P. Chronic lung disease following Stevens-Johnson syndrome. Indian Pediatr 1997; 34: 831-835.

2 Lebargy F, Wolkenstein P, Gisselbrecht M, et al. Pulmonary complications in toxic epidermal necrolysis: a prospective clinical study. Intensive Care Med 1997; 23: 1237-1244.

3 Roujeau JC, Stern RS. Severe adverse cutaneous reactions to drugs. N Engl J Med 1994; 331: 1272-1285.
4 Yatsunami J, Nakanishi Y, Matsuki H, et al. Chronic bronchobronchiolitis obliterans associated with StevensJohnson syndrome. Intern Med 1995; 34: 772-775.

5 Reyes de la Rocha S, Leonard JC, Demetriou E. Potential permanent respiratory sequela of Stevens-Johnson syndrome in an adolescent. J Adolesc Health Care 1985; 6: 220-223.

6 Sontheimer RD, Garibaldi RA, Krueger GG. StevensJohnson syndrome associated with Mycoplasma pneumoniae infections. Arch Dermatol 1978; 114: 241-244.

7 Ryu JH, Myers JL, Swensen SJ. Bronchiolar disorders. Am J Respir Crit Care Med 2003; 168: 1277-1292.

8 Epler GR. Bronchiolitis obliterans and airways obstruction associated with graft versus host disease. Clin Chest Med 1988; 9: 551-556.

9 Vogelsang GB, Lee L, Bensen-Kennedy DM. Pathogenesis and treatment of graft versus host disease after bone marrow transplant. Annu Rev Med 2003; 54: 29-52.

10 Revuz J, Penso D, Roujeau JC, et al. Toxic epidermal necrolysis. Clinical findings and prognosis factors in 87 patients. Arch Dermatol 1987; 123: 1160-1165.

11 Edwards C, Penny M, Newman J. Mycoplasma pneumonia, Stevens-Johnson syndrome, and chronic obliterative bronchitis. Thorax 1983; 38: 867-869.

12 Virant FS, Redding GJ, Novack AH. Multiple pulmonary complications in a patient with Stevens-Johnson syndrome. Clin Pediatr (Phila) 1984; 23: 412-414.

13 Wyatt SE, Nunn P, Hows JM, et al. Airways obstruction associated with graft versus host disease after bone marrow transplantation. Thorax 1984; 39: 887-894.

14 Ralph DD, Springmeyer SC, Sullivan KM, Hackman RC, Storb R, Thomas ED. Rapidly progressive air-flow obstruction in marrow transplant recipients. Possible association between obliterative bronchiolitis and chronic graft versus host disease. Am Rev Respir Dis 1984; 129: 641-644.

15 Tsunoda N, Iwanaga T, Saito T, Kitamura S, Saito K. Rapidly progressive bronchiolitis obliterans associated with Stevens-Johnson syndrome. Chest 1990; 98: 243-245.

16 Costabel U, Guzman J. Bronchoalveolar lavage in interstitial lung disease. Curr Opin Pulm Med 2001; 7: 255-261.

17 Wong DT, Weller PF, Galli SJ, et al. Human eosinophils express transforming growth factor alpha. J Exp Med 1990; 172: 673-681.

18 Zhang K, Flanders KC, Phan SH. Cellular localization of transforming growth factor-beta expression in bleomycininduced pulmonary fibrosis. Am J Pathol 1995; 147: 352-361.

19 Hoshino M, Takahashi M, Aoike N. Expression of vascular endothelial growth factor, basic fibroblast growth factor, and angiogenin immunoreactivity in asthmatic airways and its relationship to angiogenesis. J Allergy Clin Immunol 2001; 107: 295-301.

20 Lukacs NW, Hogaboam C, Chensue SW, Blease K, KunkelSL. Type 1/type 2 cytokine paradigm and the progression of pulmonary fibrosis. Chest 2001; 120: Suppl. 1, 5S-8S.

21 Trautmann A, Schmid-Grendelmeier P, Kruger K, et al. T cells and eosinophils cooperate in the induction of bronchial epithelial cell apoptosis in asthma. J Allergy Clin Immunol 2002; 109: 329-337.

22 Brito-Babapulle F. The eosinophilias, including the idiopathic hypereosinophilic syndrome. Br J Haematol 2003; 121: 203-223. 